\title{
Market Demand Elasticity and Income Inequality
}

\section{Citation}

Ibragimov, Marat and Rustam Ibragimov. 2007. Market demand elasticity and income inequality. Economic Theory 32(3): 579-587.

\section{Published Version}

http://dx.doi.org/10.1007/s00199-006-0127-1

\section{Permanent link}

http://nrs.harvard.edu/urn-3:HUL.InstRepos:2623728

\section{Terms of Use}

This article was downloaded from Harvard University's DASH repository, and is made available under the terms and conditions applicable to Other Posted Material, as set forth at http:// nrs.harvard.edu/urn-3:HUL.InstRepos:dash.current.terms-of-use\#LAA

\section{Share Your Story}

The Harvard community has made this article openly available.

Please share how this access benefits you. Submit a story.

\section{Accessibility}




\section{MARKET DEMAND ELASTICITY AND INCOME INEQUALITY*}

Marat Ibragimov $^{1}$ and Rustam Ibragimov $^{2}$

${ }^{1}$ Department of Probability Theory, Tashkent State Economics University, Tashkent, Uzbekistan

${ }^{2}$ Department of Economics, Harvard University, Littauer Center, 1875 Cambridge St., Cambridge, MA 02138,

USA (email: ribragim@fas.harvard.edu)

Summary. This paper deals with the analysis of the relation between aggregate demand for a consumption good and the distribution of income across consumers. We obtain sufficient conditions under which changes in income inequality lead to an increase or decrease in the market demand elasticities. The conditions are satisfied for individual demand functions commonly used in economic models, in particular, for the typical demand functions on luxury goods and necessities.

Keywords and phrases: income inequality, market demand, elasticity

JEL Classification Numbers: D11, D31, D63

\section{Introduction}

Several studies in the economics literature have focused on the analysis of the interrelation between income and wealth distribution and the properties of economic models. E.g., Jacobsson [21], Eichhorn, Funke and Richter [12], Thon [44], Moyes [29], [30], Arnold [3], Moyes [31] and Breton, Moyes and Trannoy [8] studied inequality reducing properties of taxation policies. Benhabib and Rustichini [6], Perotti [34], [35], Alesina and Rodrick [2], Perotti [36], Persson and Tabellini [37] and Bénabou [7], among others, have focused on the analysis of the impact of income distribution on growth. More recently, Sorger [43] analyzed the relation between the distribution of income and the level of per capita output in a deterministic one-sector growth model and endogenous labor supply. Sorger [43] showed that if the elasticity of intertemporal substitution is large, a higher output level can be achieved when the income distribution is strongly dispersed. If the elasticity of intertemporal substitution is low, the reverse relation holds. Ghiglino and Olszak-Duquenne [15] showed that the initial distribution of capital affects the dynamics in a neoclassical two-sector model with a single consumption good and endogenously determined labor supply. Ghiglino and Sorger [16] studied the effect of the initial wealth distribution on the dynamics and the determinacy of equilibria in a one sector continuous time growth model with a production externality and endogenous labor supply. Aghion [1] argued that Schumpeterian growth theory provides important insights into the relationship between growth and income inequality. Gollier [17]

\footnotetext{
*An earlier version of this paper was titled "Market demand elasticity, equilibrium stability and income inequality". The authors are grateful to an anonymous referee, Aydin Cecen and Herbert Scarf for helpful comments and suggestions. R. Ibragimov gratefully acknowledges the financial support from the Yale Graduate Fellowship and the Cowles Foundation Prize.
} 
focused on the analysis of the effects of wealth inequality on the equilibrium level of the equity premium and the risk-free rate in an Arrow-Debreu exchange economy. García-Peñalosa and Turnovsky [14] developed a canonical growth model in which the growth rate and income inequality are jointly determined and examined the effects of various structural shocks on growth and income distribution.

The notion of income inequality is closely linked to the concept of majorization ordering or the Lorenz criterion (see, e.g., Marshall and Olkin [27]). A number of studies have focused on the study of income disparity measures and applications of majorization in this and other problems of economics. The approach to the analysis of income inequality based on majorization which dates back to Lorenz [26] has been used by Atkinson [4] and Dasgupta, Sen and Starrett [9] where conditions equivalent to the Lorenz majorization criterion were obtained. Shorrocks [42] and Kakwani [22] proposed the generalized Lorenz criterion which involves weak majorization ordering instead of the majorization ordering used in [26]. More recently, Saposnik [39] stated conditions that are equivalent to the generalized Lorenz criterion. Koshevoy [23], [24] focused on the study of properties of multivariate majorization using higher dimensional generalizations of the Lorenz curve. Tsui [45] generalized the axiomatic approach to the design of income inequality measures based on the majorization criteria to a multiattribute context. Wang and Tsui [47] have focused on extensions of Gini income inequality indices using the concept of relative deprivation. Gajdos and Weymark [13] developed axiomatic characterizations of the generalized Gini social evaluation orderings in the multidimensional attributes case. Savaglio [40] examined multidimensional inequality comparisons using majorization orderings for matrices representing the distribution of commodities among people. Mitra and $\mathrm{Ok}$ [28] proposed an axiomatic characterization of income mobility orderings and measures. Basu [5] and Ok [32] analyzed fuzzy analogues of Lorenz orderings and fuzzy income inequality measures. Lapan and Hennessy [25] and Hennessy and Lapan [18] applied majorization theory to analyze the portfolio allocation problem. Recently, Ibragimov [19, 20] demonstrated that stability of conclusions of many economic models under heavy-tailedness assumptions depends crucially on majorization properties of linear combinations of thick-tailed random variables.

In this paper, we focus on the analysis of the relation between aggregate demand for a consumption good and the distribution of income across consumers. We obtain sufficient conditions under which changes in income inequality lead to an increase or decrease in the market demand elasticities. The conditions are satisfied for the typical demand functions on luxury goods and necessities.

The paper is organized as follows: Section 1 contains the definition of the majorization ordering that represents the concept of one income distribution being more uniform or more disperse than another one. In Section 2, we present the main results of the paper on the effects of income inequality on the aggregate demand. Section 3 concludes. 


\section{Basic concepts and definitions; majorization ordering}

Let there be $K$ consumers and $M$ goods in an economy. Then the function of market (aggregate) demand on $\operatorname{good} m$ is $\Phi_{m}(\mathcal{P}, \mathcal{I})=\sum_{i=1}^{K} \phi_{i m}\left(\mathcal{P}, I_{i}\right)$, where $\phi_{i m}\left(\mathcal{P}, I_{i}\right)$ is the function of the $i$ th individual's demand for the good, $\mathcal{P}=\left(p_{1}, \ldots, p_{M}\right)$ is the vector of prices on the $M$ goods in the economy and $\mathcal{I}=\left(I_{1}, \ldots, I_{K}\right)$ is the vector of incomes of the consumers.

According to the idea going back to Lorenz [26] (see also [27]), a vector $\mathcal{I}^{1}=\left(I_{1}^{1}, I_{2}^{1}, \ldots, I_{K}^{1}\right)$ represents a more uniform distribution of the total income $Y$ among $K$ consumers than a vector $\mathcal{I}^{2}=\left(I_{1}^{2}, I_{2}^{2}, \ldots, I_{K}^{2}\right)$ if $\sum_{i=1}^{l} I_{[i]}^{1} \leq \sum_{i=1}^{l} I_{[i]}^{2}, l=1, \ldots, K-1$, and $\sum_{i=1}^{K} I_{[i]}^{1}=\sum_{i=1}^{K} I_{[i]}^{2}=Y$, where $I_{i}^{j}, j=1,2$, are the income levels of the $i$ th consumer and $I_{[1]}^{j} \geq I_{[2]}^{j} \geq \ldots \geq I_{[K]}^{j}$ denote the components of the vectors $\mathcal{I}^{j}, j=1,2$, in decreasing order (if the above conditions hold, it is said that the vector $\mathcal{I}^{1}=\left(I_{1}^{1}, I_{2}^{1}, \ldots, I_{K}^{1}\right)$ is majorized by $\mathcal{I}^{2}=\left(I_{1}^{2}, I_{2}^{2}, \ldots, I_{K}^{2}\right)$, written $\left.\mathcal{I}^{1} \prec \mathcal{I}^{2}\right)$. The above definition corresponds to the intuitive understanding of one income distribution being more (or less) uniform than another one. For example, it is easy to see that the vector $(Y / N, \ldots, Y / N)$ corresponding to the case where all the total income is divided equally among the consumers is majorized by (represents a distribution which is more uniform than) any vector $\left(I_{1}, \ldots, I_{K}\right)$ with $\sum_{i=1}^{K} I_{i}=Y$. The vectors $(0, \ldots, 0, Y, 0, \ldots, 0)$ that correspond to the case where one consumer gets all the income $Y$ majorize (represent distributions which are less uniform than) any other distribution of the total income. The latter relations mean that the vectors $(Y / N, \ldots, Y / N)$ and $(Y, 0, \ldots, 0)$ are, respectively, the minimal and maximal vectors with respect to the pre-ordering relation $\prec$ on the set of income distributions $\left(I_{1}, I_{2}, \ldots, I_{K}\right)$ with $\sum_{i=1}^{K} I_{i}=Y$.

A function $f(\mathcal{I})$ is called Schur-convex (resp., Schur-concave) in $\mathcal{I}$ if $\left(\mathcal{I}^{1} \prec \mathcal{I}^{2}\right) \Longrightarrow\left(f\left(\mathcal{I}^{1}\right) \leq f\left(\mathcal{I}^{2}\right)\right.$ ) (resp. $\left(\mathcal{I}^{1} \prec \mathcal{I}^{2}\right) \Longrightarrow\left(f\left(\mathcal{I}^{1}\right) \geq f\left(\mathcal{I}^{2}\right)\right)$.

\section{Main results: income inequality and aggregate demand}

Denote by $e_{m l}(\mathcal{I})=\partial \log \Phi_{m}(\mathcal{P}, \mathcal{I}) / \partial \log p_{l}$ the cross-price elasticity of the aggregate demand on the $m$-th good with respect to the $l$-th good's price. The quantity $e_{m m}(\mathcal{I})=\partial \log \Phi_{m}(\mathcal{P}, \mathcal{I}) / \partial \log p_{m}$ is the own-price elasticity of the aggregate demand on the $m$-th good.

The results in this section of the paper provide conditions under which the functions $e_{m l}(\mathcal{I})$ are Schur-convex or Schur-concave in $\mathcal{I}$. That is, they provide conditions under which the functions $\left|e_{m l}(\mathcal{I})\right|$ and $-\left|e_{m l}(\mathcal{I})\right|, m, l=$ $1, \ldots, M$, preserve the majorization relation $\prec$, that is, under which $\mathcal{I}^{1} \prec \mathcal{I}^{2}$ implies that $\left|e_{m l}\left(\mathcal{I}^{1}\right)\right| \leq\left|e_{m l}\left(\mathcal{I}^{2}\right)\right|$ or $\left|e_{m l}\left(\mathcal{I}^{2}\right)\right| \leq\left|e_{m l}\left(\mathcal{I}^{1}\right)\right|$. Using the above property of minimality and maximality of the vectors $(Y / N, \ldots, Y / N)$ and $(Y, 0, \ldots, 0)$, we use the results to obtain estimates for the elasticities.

By [27, 3.A.5], the function $e_{m l}(\mathcal{I})=\partial \log \Phi_{m}(\mathcal{P}, \mathcal{I}) / \partial \log p_{l}$ is Schur-concave in $\mathcal{I}$ if and only if it is symmetric in $\mathcal{I}$ and, for fixed $\tilde{\mathcal{I}}=\left(I_{3}, \ldots, I_{n}\right)$, the function $e_{m l}\left(I_{1}, I_{2}, \tilde{\mathcal{I}}\right)$ is Schur-concave in $I_{1}$ and $I_{2}$, that is, if and only if the function $h(I, \lambda, \tilde{\mathcal{I}})=e_{m l}(I-\lambda, \lambda, \tilde{\mathcal{I}})$ is decreasing in the parameter $\lambda \in[0, I / 2]$ that represents a mean-preserving spread in the income distribution. If $\Phi_{m}(\mathcal{P}, \mathcal{I})$ is differentiable in $\mathcal{I}$, we thus 
obtain that $e_{m l}(\mathcal{I})$ is Schur-concave in $\mathcal{I}$ if and only if $\frac{\partial^{2} \log \Phi_{m}(I-\lambda, \lambda, \tilde{\mathcal{I}})}{\partial \log p_{l} \partial \lambda} \leq 0$, that is, if and only if the function $\Phi_{m}(\mathcal{P}, I-\lambda, \lambda, \tilde{\mathcal{I}})$ is $\log$-submodular in $p_{l}$ and $\lambda$. Similarly, the function $e_{m l}(\mathcal{I})$ is Schur-convex in $\mathcal{I}$ if and only if $\frac{\partial^{2} \log \Phi_{m}(I-\lambda, \lambda, \tilde{\mathcal{I}})}{\partial \log p_{l} \partial \lambda} \geq 0$, that is, if and only if the function $\Phi_{m}(\mathcal{P}, I-\lambda, \lambda, \tilde{\mathcal{I}})$ is $\log$-supermodular in $p_{l}$ and $\lambda .{ }^{1}$

The results in this section show that Schur-concavity of the aggregate demand $\Phi_{m}$ in $I$ holds if a meanpreserving spread in the income distribution leads to an upward shift of the demand function and to a decrease in its slope. Schur-convexity of $\Phi_{m}$ in $I$ holds if the demand function shifts downward and its slope increases with a mean-preserving spread. ${ }^{2}$ Example 1 below shows that an increase in income inequality leads to a decrease in the absolute value of the elasticity of the typical demand on luxuries and to an increase in the absolute value of the elasticity of the typical demand on necessities.

Suppose that

$$
\phi_{1 m}(\mathcal{P}, I)=\phi_{2 m}(\mathcal{P}, I)=\ldots=\phi_{K m}(\mathcal{P}, I)=\phi_{m}(\mathcal{P}, I)
$$

Denote by $S_{1} \subset \mathbf{R}^{n+1}$ the domain of definition of the function $\phi_{m}(\mathcal{P}, I)$ and by $S_{2}=\left\{(\mathcal{P}, \mathcal{I})=\left(\mathcal{P}, I_{1}, \ldots, I_{K}\right) \in\right.$ $\left.\mathbf{R}^{M+K}:\left(\mathcal{P}, I_{i}\right) \in S_{1}, i=1, \ldots, K\right\}$ the domain of definition of the function $\Phi_{m}(\mathcal{P}, \mathcal{I})$.

Theorem 1 Let goods $m$ and $l$ be complements. Then the following conclusions hold.

(i) If the functions $\phi_{m}(\mathcal{P}, I)$ and $\partial \phi_{m}(\mathcal{P}, I) / \partial p_{l}$ are convex with respect to $I$ for $(\mathcal{P}, I) \in S_{1}$, then the conditions $\left(\mathcal{P}, \mathcal{I}^{1}\right),\left(\mathcal{P}, \mathcal{I}^{2}\right) \in S_{2}$ and $\mathcal{I}^{1} \prec \mathcal{I}^{2}$ imply $\left|e_{m l}\left(\mathcal{I}^{1}\right)\right| \geq\left|e_{m l}\left(\mathcal{I}^{2}\right)\right|$. That is, the more non-uniform is the distribution of the total income among consumers in the economy, the smaller is the cross-price elasticity of the demand on the $m$-th good with respect to the $l$-th good's price in absolute value.

(ii) If the functions $\phi_{m}(\mathcal{P}, \mathcal{I})$ and $\partial \phi_{m}(\mathcal{P}, \mathcal{I}) / \partial p_{l}$ are concave with respect to $I$ for $(\mathcal{P}, I) \in S_{1}$, then $\left(\mathcal{P}, \mathcal{I}^{1}\right)$, $\left(\mathcal{P}, \mathcal{I}^{2}\right) \in S_{2}$ and $\mathcal{I}^{1} \prec \mathcal{I}^{2}$ imply $\left|e_{m l}\left(I^{1}\right)\right| \leq\left|e_{m l}\left(I^{2}\right)\right|$. That is, the more non-uniform is the distribution of the total income among consumers in the economy, the larger is the cross-price elasticity of the demand on the $m$-th good with respect to the l-th good's price in absolute value.

Proof. According to Theorem 3.C.1 in [27], from convexity of the functions $\phi_{m}(\mathcal{P}, I)$ and $\partial \phi_{m}(\mathcal{P}, I) / \partial p_{l}$ in $I$ it follows that the functions $\Phi_{m}(\mathcal{P}, \mathcal{I})=\sum_{i=1}^{K} \phi_{m}\left(\mathcal{P}, I_{i}\right)$ and $\partial \Phi_{m}(\mathcal{P}, \mathcal{I}) / \partial p_{l}=\sum_{i=1}^{K} \partial \phi_{m}\left(\mathcal{P}, I_{i}\right) / \partial p_{l}$ are Schur-convex in $\mathcal{I}$ on $S_{2}$, that is, $\left(\mathcal{P}, \mathcal{I}^{1}\right),\left(\mathcal{P}, \mathcal{I}^{2}\right) \in S_{2}$ and $\mathcal{I}^{1} \prec \mathcal{I}^{2}$ imply $\Phi_{m}\left(\mathcal{P}, \mathcal{I}^{1}\right) \leq \Phi_{m}\left(\mathcal{P}, \mathcal{I}^{2}\right)$ and $\partial \Phi_{m}\left(\mathcal{P}, \mathcal{I}^{1}\right) / \partial p_{l} \leq \partial \Phi_{m}\left(\mathcal{P}, \mathcal{I}^{2}\right) / \partial p_{l}$. From these relations it follows that

$$
\left.e_{m l}\left(\mathcal{I}^{1}\right)=\left[\left(\partial \Phi_{m}\left(\mathcal{P}, \mathcal{I}^{1}\right) / \partial p_{l}\right) p_{l}\right] / \Phi_{m}\left(\mathcal{P}, \mathcal{I}^{1}\right) \leq\left[\partial \Phi_{m}\left(\mathcal{P}, \mathcal{I}^{2}\right) / \partial p_{l}\right) p_{l}\right] / \Phi_{m}\left(\mathcal{P}, \mathcal{I}^{2}\right)=e_{m l}\left(\mathcal{I}^{2}\right)
$$

as $\mathcal{I}^{1} \prec \mathcal{I}^{2}$. Since the goods $m$ and $l$ are complements, we get that $\left|e_{m l}\left(\mathcal{I}^{1}\right)\right| \geq\left|e_{m l}\left(\mathcal{I}^{2}\right)\right|$ as $\mathcal{I}^{1} \prec \mathcal{I}^{2}$, that is, part (i) of the theorem holds. Part (ii) of the theorem may be proven in complete similarity.

\footnotetext{
${ }^{1} \mathrm{Sub}$ - and supermodular functions are also referred to as, respectively, $L$-sub- and $L$-superadditive functions; the reader is referred to, among other works, Chapter 6 in [27] for a review of basic properties and examples of such functions.

${ }^{2}$ According to the discussion in Section 3, the demand shifts in response to income inequality changes are observed, for instance, for food commodities.
} 
In the case when goods $m$ and $l$ are substitutes, the conclusions of Theorem 1 are correspondingly reversed.

Theorem 1 implies the following corollary for the own-price elasticity of the market demand on a good.

Corollary 1 (i) If the functions $\phi_{m}(\mathcal{P}, I)$ and $\partial \phi_{m}(\mathcal{P}, I) / \partial p_{m}$ are convex with respect to $I$ for $(\mathcal{P}, I) \in S_{1}$, then the conditions $\left(\mathcal{P}, \mathcal{I}^{1}\right),\left(\mathcal{P}, \mathcal{I}^{2}\right) \in S_{2}, \mathcal{I}^{1} \prec \mathcal{I}^{2}$ imply $\left|e_{m m}\left(\mathcal{I}^{1}\right)\right| \geq\left|e_{m m}\left(\mathcal{I}^{2}\right)\right|$. That is, the more non-uniform is the distribution of the total income among consumers in the economy, the smaller is the own-price elasticity of the demand on the $m$-th good in absolute value.

(ii) If the functions $\phi_{m}(\mathcal{P}, I)$ and $\partial \phi_{m}(\mathcal{P}, I) / \partial p_{m}$ are concave with respect to $I$ for $(\mathcal{P}, I) \in S$, then the conditions $\left(\mathcal{P}, \mathcal{I}^{1}\right),\left(\mathcal{P}, \mathcal{I}^{2}\right) \in S_{2}$ and $\mathcal{I}^{1} \prec \mathcal{I}^{2}$ imply $\left|e_{m m}\left(\mathcal{I}^{1}\right)\right| \leq\left|e_{m m}\left(\mathcal{I}^{2}\right)\right|$. That is, the more non-uniform is the distribution of the total income among consumers in the economy, the larger is the own-price elasticity of the demand on the $m$-th good in absolute value.

Using the above property that $\mathcal{Y}_{1}=(Y / N, \ldots, Y / N) \prec\left(I_{1}, \ldots, I_{K}\right) \prec \mathcal{Y}_{2}=(Y, 0, \ldots, 0)$ for all income distributions $\left(I_{1}, \ldots, I_{K}\right)$ with $\sum_{i=1}^{K} I_{i}=Y$, we obtain the following inequalities for the absolute value of the own-price elasticity of market demand.

Corollary 2 (i) If the functions $\phi_{m}(\mathcal{P}, I)$ and $\partial \phi_{m}(\mathcal{P}, I) / \partial p_{m}$ are convex with respect to $I$ for $(\mathcal{P}, I) \in S_{1}$ and $\left(\mathcal{P}, \mathcal{Y}_{j}\right) \in S_{2}, j=1,2$, then $\left|e_{m m}\left(\mathcal{Y}_{1}\right)\right| \geq\left|e_{m m}(I)\right| \geq\left|e_{m m}\left(\mathcal{Y}_{2}\right)\right|$ for all income distributions $\mathcal{I}=\left(I_{1}, \ldots, I_{K}\right)$ with $\sum_{i=1}^{K} I_{i}=Y$.

(ii) If the functions $\phi_{m}(\mathcal{P}, I)$ and $\partial \phi_{m}(\mathcal{P}, I) / \partial p_{m}$ are concave with respect to $I$ for $(\mathcal{P}, I) \in S_{1}$ and $\left(\mathcal{P}, \mathcal{Y}_{j}\right) \in$ $S_{2}, j=1,2$, then $\left|e_{m m}\left(\mathcal{Y}_{1}\right)\right| \leq\left|e_{m m}(I)\right| \leq\left|e_{m m}\left(\mathcal{Y}_{2}\right)\right|$ for all income distributions $\mathcal{I}=\left(I_{1}, \ldots, I_{K}\right)$ with $\sum_{i=1}^{K} I_{i}=$ $Y$.

Example 1. Let the individual demand function on the $m$-th good in (1) be a typical function of demand on luxuries $\phi_{m}\left(p_{m}, I\right)=\alpha I\left(I-\gamma p_{m}\right) /\left(I+\beta p_{m}\right), \alpha>0, \beta>0, \gamma \geq 0$. It is not difficult to verify that the function $\phi_{m}\left(p_{m}, I\right)$ satisfies the conditions of part (i) of Corollary 1 on the set $S_{1}=\left\{\left(p_{m}, I\right): I>\beta p_{m} / 2\right\}$. Similarly, if the individual demand function on the $m$-th good is a typical demand function on necessities $\phi_{m}\left(p_{m}, I\right)=\alpha\left(I-\gamma p_{m}\right) /\left(I+\beta p_{m}\right), \alpha>0, \beta>0, \gamma \geq 0$, then it satisfies the conditions of Part (ii) of Corollary 1 on $S_{1}=\left\{\left(p_{m}, I\right): I>2 \beta p_{m}\right\}$.

Example 2. The analysis of Schur-convexity and Schur-concavity of the demand elasticities $e_{m l}(\mathcal{I})$ in $\mathcal{I}$ simplifies in the case when the aggregate demand $\Phi_{m}(\mathcal{P}, \mathcal{I})$ has the form of the almost ideal demand system introduced in [10] (see relations (8"), (13) and (8"') in that work). In the notations of this paper, the almost ideal demand system corresponds to the aggregate demand functions $\Phi_{m}(\mathcal{P}, \mathcal{I})$ in the following form:

$\Phi_{m}(\mathcal{P}, \mathcal{I})=Y\left[\alpha_{m}+\sum_{j=1}^{M} \gamma_{m j} \log p_{j}+\beta_{m}\left(\frac{\sum_{i=1}^{K} I_{i} \log I_{i}}{Y}-\alpha_{0}-\sum_{k=1}^{M} \alpha_{k} \log p_{k}-\frac{1}{2} \sum_{j=1}^{M} \sum_{k=1}^{M} \gamma_{k j} \log p_{k} \log p_{j}\right)\right]$

where $Y=\sum_{i=1}^{K} I_{i}$ and $\alpha_{i}, \beta_{i}$ and $\gamma_{i j}$ are parameters such that $\sum_{k=1}^{M} \alpha_{k}=1, \sum_{i=1}^{M} \gamma_{i j}=\sum_{j=1}^{M} \gamma_{i j}=\sum_{k=1}^{M} \beta_{k}=$ 
$0, \gamma_{i j}=\gamma_{j i}{ }^{3}$ Since the entropy $E(\mathcal{I})=-\sum_{i=1}^{K}\left(I_{i} / Y\right) \log \left(I_{i} / Y\right)$ is Schur-concave in $\mathcal{I}=\left(I_{1}, \ldots, I_{K}\right)$ (see, e.g., $[27,3 . D .1])$, we obtain that Schur-convexity and Schur-concavity of $\Phi_{m}(\mathcal{P}, \mathcal{I})$ is determined by the sign of the coefficient $\beta_{m}$. Namely, $\Phi_{m}(\mathcal{P}, \mathcal{I})$ is Schur-convex in $\mathcal{I}$ if $\beta_{m}>0$ and is Schur-concave in $\mathcal{I}$ if $\beta_{m}<0$. Since

$$
e_{m l}(\mathcal{I})=\frac{\partial \log \Phi_{m}(\mathcal{P}, \mathcal{I})}{\partial \log p_{l}}=\frac{Y\left(\gamma_{m l}-\beta_{m} \alpha_{l}-\sum_{k=1}^{M} \gamma_{k l} \log p_{k}\right)}{\Phi_{m}(\mathcal{P}, \mathcal{I})}
$$

the above implies that $\left|e_{m l}(\mathcal{I})\right|$ is Schur-convex in $\mathcal{I}$ if goods $m$ and $l$ are substitutes and $\beta_{m}<0$ or if goods $m$ and $l$ are complements and $\beta_{m}>0$. Similarly, from the above analysis we get that $\left|e_{m l}(\mathcal{I})\right|$ is Schur-concave in $\mathcal{I}$ if goods $m$ and $l$ are substitutes and $\beta_{m}>0$ or if goods $m$ and $l$ are complements and $\beta_{m}<0$. In particular, the own-price elasticity of the market demand on the $m$-th good $e_{m m}(\mathcal{I})=\partial \log \Phi_{m}(\mathcal{P}, \mathcal{I}) / \partial \log p_{m}$ is Schur-convex in $\mathcal{I}$ if $\beta_{m}>0$ and is Schur-convex in $\mathcal{I}$ if $\beta_{m}<0$.

\section{Conclusion and suggestions for further research}

In this paper, we focused on the analysis of the relation between the aggregate demand for a consumption good and income distribution across consumers. The results in the paper provide sufficient conditions under which changes in income distribution lead to an increase or decrease in the market demand elasticities. The conditions concern a shift in the individual demand functions in response to income redistribution, and a change in their slope.

The empirical study in [46] suggests that greater equality in income distribution reduces the average meat consumption (the effect is negative for red meats as well as for poultry). ${ }^{4}$ On the other hand, according to the empirical results for the population of Cali, Colombia, obtained by Pinstrup-Andersen and Caicedo [38], reduction in income inequality has a considerable positive impact on the demand for food commodities, including meat. Senauer [41] reports that the lower-income households are more price responsive for the consumption of rice in developing countries. However, the analysis of the U.S. data on food commodities and household poverty status in [33] provides estimates for own-price elasticities that are similar between the income strata. In view of the results in this paper, further analysis is desirable to explain the disparities in the above empirical conclusions.

The results obtained in the paper can be used, together with the majorization results for linear combinations of thick-tailed random variables obtained in $[19,20]$, to study the robustness of economic models to both inequality and heavy-tailedness in income distribution. The areas of other possible applications include, in particular, studies of asset pricing, where the majorization-based analysis seems to naturally complement the approach based on the concept of mean-preserving spread discussed by, e.g., Gollier [17]. ${ }^{5}$

\footnotetext{
${ }^{3}$ One can also consider, as in [10], generalizations of (1) in which the expression $\sum_{i=1}^{K} I_{i} \log I_{i}$ is replaced by $\sum_{i=1}^{K} I_{i} \log \left(I_{i} / k_{i}\right)$ and the parameters $k_{i}$ can be interpreted as a sophisticated measure of household size that could account, in particular, for age composition and other household characteristics.

${ }^{4}$ See also Eales and Unnevehr [11] for estimates of demand systems for meat products.

${ }^{5}$ We thank an anonymous referee for drawing our attention to this reference and to the almost ideal demand system [10] discussed in Example 3.
} 


\section{References}

[1] Aghion, P.: Schumpeterian growth theory and the dynamics of income inequality. Econometrica 70, $855-882(2002)$

[2] Alesina, A., Rodrik, D.: Distributive politics and economic growth. Quarterly Journal of Economics 109, 465-490 (1994)

[3] Arnold, B. C.: The Lorenz order and the effects of taxation policies. Bulletin of Economic Research 42, 249-264 (1990)

[4] Atkinson, A. B.: On the measurement of inequality. Journal of Economic Theory 5, 244-263 (1970)

[5] Basu, K.: Axioms for a fuzzy measure of inequality. Mathematical Social Sciences 14, 275-288 (1987)

[6] Benhabib, J., Rustichini, A.: Social conflict and inequality. Working paper, New York University (1991)

[7] Bénabou, R.: Inequality and growth. In: NBER Macroeconomics Annual (B. Bernanke and J. Rotemberg, eds.), 11-74 (1996)

[8] Breton, M. L., Moyes, P., Trannoy, A.: Inequality reducing properties of composite taxation. Journal of Economic Theory 69, 71-103 (1996)

[9] Dasgupta, P. A., Sen, A., Starrett, D.: Notes on the measurement of inequality. Journal of Economic Theory 6, 180-187 (1973)

[10] Deaton, A., Muellbauer, J.: An almost ideal demand system. American Economic Review 70, 312-326 (1980)

[11] Eales, J. S., Unnevehr, L. J.: Demand for beef and chicken products: separability and structural change. American Journal of Agricultural Economics 70, 521-532 (1988)

[12] Eichhorn, W., Funke, H., Richter, W. F.: Tax progression and inequality of income distribution. Journal of Mathematical Economics 13, 127-131 (1984)

[13] Gajdos, T., Weymark, J. A.: Multidimensional generalized Gini indices. Economic Theory 26, 471-496 $(2005)$

[14] García-Peñalosa, C., Turnovksy, S. J.: Growth and income inequality: a canonical model. Economic Theory 28, 25-49 (2006)

[15] Ghiglino, C., Olszak-Duquenne, M.: Inequalities and fluctuations in a dynamic general equilibrium model. Economic Theory 17, 1-24 (2001) 
[16] Ghiglino, C., Sorger, G.: Poverty traps, indeterminacy, and the wealth distribution. Journal of Economic Theory 105, 120-139 (2002)

[17] Golier, C.: Wealth inequality and asset pricing. Review of Financial Studies 68, 181-203 (2001)

[18] Hennessy, D. A., Lapan, H. E.: An algebraic theory of portfolio allocation. Economic Theory 22, 193-210 (2003)

[19] Ibragimov, R.: On the robustness of economic models to heavy-tailedness assumptions. Mimeo, Yale University (2004). Available at http://post.economics.harvard.edu/faculty/ibragimov/Papers/HeavyTails.pdf

[20] Ibragimov, R.: New majorization theory in economics and martingale convergence results in econometrics. Ph.D. dissertation, Yale University (2005)

[21] Jacobsson, U.: On the measurement of the degree of progression. Journal of Public Economics 8, 161-168 (1976)

[22] Kakwani, N.: Welfare ranking of income distributions. In: Advances in econometrics (R. L. Basmann and G. F. Rhodes, Jr., eds.) 3. JAI Press, Greenwich, CT 1984

[23] Koshevoy, G.: Multivariate Lorenz majorization. Social Choice and Welfare 12, 93-102 (1995)

[24] Koshevoy, G.: The Lorenz zonotope and multivariate majorizations. Social Choice and Welfare 15, 1-14 (1998)

[25] Lapan, H. E., D. A. Hennessy: Symmetry and order in the portfolio allocation problem. Economic Theory 19, 747-772 (2002)

[26] Lorenz, M. O.: Methods of measuring concentration of wealth. Journal of the American Statistical Association 9, 209-219 (1905)

[27] Marshall, A. W., Olkin, I.: Inequalities: Theory of Majorization and Its Applications. New York: Academic Press 1979

[28] Mitra, T., Ok, E.: The measurement of income mobility: A partial ordering approach. Economic Theory 12, 77-102 (1998)

[29] Moyes, P.: Equiproportionate growth of incomes and after-tax inequality. Bulletin of Economic Research 41, 287-294 (1989)

[30] Moyes, P.: Some classes of functions that preserve the inequality and welfare orderings of income distributions. Journal of Economic Theory 49, 347-359 (1989) 
[31] Moyes, P.: Inequality reducing and inequality preserving transformations of incomes: symmetric and individualistic transformations. Journal of Economic Theory 63, 271-298 (1994)

[32] Ok, E. A.: Fuzzy measurement of income inequality: Some possibility results on the fuzzification of the Lorenz ordering. Economic Theory 7, 513-530 (1996)

[33] Park, J. L., Hocomb, R. B., Raper, K. C., Capps, O.: A demand systems analysis of food commodities by U.S. households segmented by income. American Journal of Agricultural Economics 78, 290-300 (1996)

[34] Perotti, R.: Income distribution, politics and growth. American Economic Review 82, 311-316 (1992)

[35] Perotti, R.: Political equilibrium, income distribution, and growth. Review of Economic Studies 60, $755-776(1993)$

[36] Perotti, R.: Income distribution and investment. European Economic Review 38, 827-835 (1994)

[37] Persson, T., Tabellini, G.: Is inequality harmful for growth? American Economic Review 84, 600-621 (1994)

[38] Pinstrup-Andersen, P., Cailedo, E.: The potential impact of changes in income distribution on food demand and human nutrition. American Journal of Agricultural Economics 60, 402-415 (1978)

[39] Saposnik, R.: A note on majorization theory and the evaluation of income distributions. Economics Letters 42, 179-183 (1993)

[40] Savaglio, E.: Multidimensional inequality with variable population size. Economic Theory 28, 85-94 (2006)

[41] Senauer, B.: Household behavior and nutrition in developing countries. Food policy 15, $408-417$ (1990)

[42] Shorrocks, A. F.: Ranking income distributions. Economica 50, 3-17 (1983)

[43] Sorger, G.: Income and wealth distribution in a simple model of growth. Economic Theory 16, 23-42 $(2000)$

[44] Thon, D.: Redistributive properties of progressive taxation. Mathematical Social Sciences 14, 185-191 (1987)

[45] Tsui, K.-y.: Multidimensional inequality and multidimensional generalized entropy measures: an axiomatic derivation. Social Choice and Welfare 16, 145-157 (1999)

[46] Unnevehr, L. J., Khoju, M.: Economic development, income distribution and meat demand. Journal of International Food \& Agribusiness Marketing 2 (3/4) (1991)

[47] Wang, Y-q., Tsui, K.-y.: A new class of deprivation-based generalized Gini indices. Economic Theory 16, 363-377 (2000) 